\title{
The novel coronavirus infection pneumonia in Zhejiang province, China: early transmission dynamics, the government measures, and current situation of diagnosis and treatment
}

\section{Shen junwen}

the department of urology, the first hospital of Huzhou

\section{Chen yu}

the department of urology, the first hospital of Huzhou

\section{Tang jianer}

the department of urology, the first hospital of Huzhou

Wang rongjiang ( $\sim$ wrj688765@163.com)

the department of urology, the first hospital of Huzhou

\section{Fang zhihai}

the department of urology, the first hospital of Huzhou

\section{Yao jianxiang}

the department of urology, the first hospital of Huzhou

\section{Liu bingyao}

the school of nursing, Huzhou university

\section{Zhou ying}

the department of urology, the first hospital of Huzhou

\section{shi zhanqing}

the department of nephrology, the first hospital of Huzhou

\section{Research Article}

Keywords: COVID-19, novel coronavirus infection pneumonia, Zhejiang province, transmission dynamics

Posted Date: March 26th, 2020

DOI: https://doi.org/10.21203/rs.3.rs-19540/v1

License: (c) (i) This work is licensed under a Creative Commons Attribution 4.0 International License.

Read Full License 


\section{Abstract}

Objective: to analyze the early transmission dynamics, the government measures, and current situation of diagnosis and treatment for novel coronavirus infection pneumonia in Zhejiang province, china.

Methods: We collected the daily number of newly confirmed novel coronavirus infection pneumonia (NCP) patients and the number of discharge patients by February 8 in Zhejiang province. We analyzed the characteristics, exposure history, and the clinical symptoms of NCP patients.

Results: there were 1075 confirmed NCP patients and 173 discharge patients in Zhejiang province by February 8 . The daily number of newly confirmed NCP patients got decreased since January 29 (27 patients on February 8), while the daily number of newly discharge NCP patients was increasing (46 patients on February 8). Before February 1, the imported NCP patients contained the most significant part of total NCP patients. And the local infection of NCP patients occupied the main reason. 77 patients needed to stay in ICU. 26 of 77 patients had greater life danger. Fortunately, no patient was dead, and no health care worker got the infection. At the same time, the government of Zhejiang province strictly restricted the movement of people to prevent the NCP from further spread.

Conclusion: The early spread of NCP in Zhejiang province was speedy. After the government of Zhejiang province took strict measures to restrict the movement of people, the difficult situation of NCP got noticeable relief in Zhejiang province since February 1.

\section{Introduction}

The novel coronavirus infection pneumonia (2019-nCOV) was reported to appear in the city of Wuhan, the provincial city of Hubei province, in December $2019^{(1,2)}$. A few early kinds of research pointed out that the basic reproductive number of NCIP was more significant than $2^{(3,4)}$. And another study of 41 confirmed 2019-nCOV patients revealed the clinical symptom of the 2019-nCOV, and the fatality rate of the 2019$\mathrm{nCOV}$ was $15 \%$ in this research ${ }^{(5)}$. At the same time, Italy, Germany, and the united states had found the 2019-nCOV patients in January $2020^{(6,7)}$. The information represented the strong infectivity of 2019nCOV.

The Chinese government took the emergency response level 1 to the 2019-nCOV on January 15 and named this novel coronavirus infection pneumonia as NCP. Every district of china, including the Zhejiang province, had emergency measures in the following days. Since February 8 , there were 33738 confirmed NCP patients in china and 1075 confirmed NCP patients in Zhejiang province. The number of confirmed NCP patients in Zhejiang province was second to the Hubei province in China.

To analyze the early transmission dynamics of NCP, the government measures for NCP, and the current situation of treatment for NCP patients in Zhejiang province, we did the research and wrote this paper.

\section{Methods}




\section{sources of data}

We obtained the number of NCP patients' data released by the Zhejiang health commission from January 20 to February 8 (Zhejiang province found the first confirmed NCP patients on January 20). And we collected the government response measures to the disease of NCP from the Zhejiang government's announcement.

\section{diagnostic criteria for NCP patients}

A confirmed NCP patient was diagnosed with respiratory or blood specimens that tested positive for NCP by at least one of the following two methods: 1 . at least two positive results by real-time reversetranscription-polymerase chain-reaction (RT-PCR) assay for NCP; 2 . a genetic sequence that matches $\mathrm{NCP}{ }^{(8)}$. The above standard confirmed all the NCP patients in Zhejiang province. And Zhejiang health commission released the daily new diagnosis of NCP patients.

\section{the collection information and criteria}

We collected the government response measures to the disease of NCP through the Zhejiang government's announcement from January 20 to February 8 . And we would arrange the detail of actions in the part of the results. The value of steps was analyzed in the part of the discussion.

We recorded the details of NCP patients in Zhejiang province that were released by the Zhejiang health commission from January 20 to February 8.

We recorded the number of accumulative confirmed NCP patients, the daily number of newly diagnosed NCP patients, the accumulated amount of discharge NCP patients, the daily number of discharge NCP patients. The patients staying in ICU were paid more attention.

We recorded the epidemiological history of all the confirmed NCP patients. We divided the patients' epidemiological history into four types: 1. from Hubei province: the patients travel from the Hubei province in the past 14 days; 2 . from other area: the patients travel from other cities in China (it also contained that the patient might travel from other cities in Zhejiang province) or other countries in the past 14 days; 3 . local infection: the patients did not have the travel history but had the contact history of the confirmed or suspected NCP patients in the local area in the past 14 days; 4 . unknown: the patients did neither have the travel history nor the contact history of the confirmed or suspected NCP patients in the local area in the past 14 days. The imported NCP patients contained the types of from Hubei province and other regions. 
We recorded the clinical symptoms of all the confirmed NCP patients. According to the announcement of Zhejiang health commission, we recorded the following clinical symptoms: fever, respiratory symptoms (it contained cough, expectoration, and pharyngalgia), digestive tract symptoms (it contained stomachache, diarrhea, nausea, and emesis), muscle and neurological symptoms (it contained headache, muscular soreness, and lacked in strength), abnormal changes in chest CT, or without clinical symptoms. The number of the above symptoms was counted. And we would show the data in the part of the results.

We recorded the time from clinical signs to NCP diagnosis for every confirmed NCP patient from the announcement of the Zhejiang health commission. For example, zero-day might mean that this patient's time from clinical symptoms to NCP diagnosis was less than 24 hours. And two days might suggest that this patient's time from clinical signs to NCP diagnosis was less than 72 hours but more massive than 48 hours.

We paid attention to the condition of whether the health care worker had got the infection of NCP and recorded the number of health care workers with NCP.

\section{statistical analysis}

For the characteristics of NCP patients, we used SPSS 25.0 to analyze the data average and mean deviation.

\section{ethics approval}

This study was approved by the ethics committee of the first hospital of Huzhou. And the waiver of informed consent for the study was obtained from the ethics committee of the first hospital of Huzhou.

\section{Results}

with the rapid outbreak of NCP in Zhejiang province in late January 2020, the local government of Zhejiang province had taken four measures to protect the NCP to further diffusion in Zhejiang province: 1. Zhejiang had made the emergency response level 1 to the NCP (January 23, 2020); 2 . Zhejiang shut down unnecessary public facilities in the cities and canceled all kinds of parties(January 24-26, 2020$) ; 3$. the people from the Chinese other province were forbidden to enter into Zhejiang province (January 28-30, 2020); 4. The residents were restricted from going outside (February $3-4,2020)$. The detail measures can be seen in the supplement table 1. By the time of writing this paper (February 10, 2020), the above measures are still being implemented. 
the first patient of NCP in the Zhejiang province was found on January 20. however, 1075 patients had been diagnosed with NCP in Zhejiang province on February 8. Among the Chinese 31 regions, the number of NCP patients in Zhejiang province ranked second only to Hubei province. Among the 1075 NCP patients, 77 critical patients needed intensive care. In fig 1, we demonstrated the number of accumulative confirmed NCP patients and accumulative discharge NCP patients from January 20 to February 8.173 patients had got recovery and left the hospital. And no patient was dead. In fig 2, we demonstrated the daily number of newly confirmed NCP patients and the daily number of discharge NCP patients. From fig 2 , there were two important information: 1 . the peak time for newly confirmed NCP patients was January 29. And the number of freshly established NCP patients was decreased since January 29; 2 . there was the first time on February 8 that the amount of discharge NCP patients was more significant than the number of newly diagnosed NCP cases. If we can keep the tendency, we had the confidence to conquer the NCP. In fig 3, we demonstrated the information of patients in ICU from January 23 to February 8. From fig 3, the situation for the critical patients was worried: 1 . the number of patients who needed to stay in ICU was still increasing; 2 . the number of NCP patients whose life danger in ICU was also growing. Although there was no dead patient in the Zhejiang province right now, those NCP patients in ICU were still facing the threat of death.

According to the government circular of Zhejiang province, we could get 982 of 1075 NCP patients' epidemiological history (part patients' epidemiological history was not released before January 28). There were 490 imported NCP patients (417 patients traveled from Hubei province, 72 patients traveled from other Chinese cities or other countries, and 1 patient reached Hubei province and other areas in the past 14 days). There were 419 local NCP infection patients and 73 patients with an unknown epidemiological history. In fig 4, the daily number of newly diagnosed NCP patients' epidemiological history was demonstrated. And in fig 5-6, the daily percentage of newly diagnosed NCP patients' epidemiological history was demonstrated. From fig 5 , we can easily find that the rate of imported NCP patients from Hubei province got decreased. In contrast, local infection NCP patients became the main people for newly diagnosed NCP patients in the past week. And fig 6 showed the percentage of imported NCP patients from other areas got increased. And the number of newly diagnosed NCP patients with unknown epidemiological history could not be ignored. Those variations tendencies in fig 6 reflected that the disease of NCP had spread from Hubei province to other areas in our country and the whole world.

According to the government circular of Zhejiang province, we could get 982 of 1075 NCP patients' age, sexy, clinical symptoms, and the time from clinical signs to NCP diagnosis (part patients' information were not released before January 28). There were 492 male patients and 490 female patients. There were 3 patients whose birth time was smaller than 12 months. And there were 15 patients whose ages were older than 80 years. The median age of 982 patients was $46.43 \pm 15.21$ years. 654 patients had a fever. 400 patients had respiratory symptoms. 119 patients had digestive tract symptoms. 32 patients had 
headaches or muscular soreness or lacked in strength. 237 patients had abnormal changes in chest CT. And 117 patients did not have clinical symptoms. The media time of 982 patients from clinical symptoms to NCP diagnosis was $5.48 \pm 4$ days. In table 1, we demonstrated the detail of NCP patients at different times. In the supplement table 2, we demonstrated the detail of NCP patients between with different epidemiological history.

Table 1: characteristics of NCP patients in zhejiang province between different time.

\begin{tabular}{|l|l|l|}
\hline & $\begin{array}{l}\text { January 28-January 31 } \\
(\mathrm{n}=426)\end{array}$ & $\begin{array}{l}\text { February 1-February 8 } \\
(\mathrm{n}=476)\end{array}$ \\
\hline Sexy (male/female) & $225 / 201$ & $227 / 249$ \\
\hline Media age (y) & $44.4 \pm 14.5$ & $48.3 \pm 15.9$ \\
\hline$<16 \square \mathrm{n}, \% \square$ & $12,2.8$ & $12,2.5$ \\
\hline $16-45 \square \mathrm{n}, \% \square$ & $210,49.3$ & $188,39.5$ \\
\hline $46-60 \square \mathrm{n}, \% \square$ & $151,35.4$ & $167,35.1$ \\
\hline$>60 \square \mathrm{n}, \% \square$ & $53,12.4$ & $109,22.9$ \\
\hline clinical symptoms (n) & & 308 \\
\hline fever & 296 & 179 \\
\hline respiratory symptoms & 177 & 71 \\
\hline digestive tract symptoms & 33 & 16 \\
\hline $\begin{array}{l}\text { headache or } \\
\text { soreness or lacked in strength }\end{array}$ & 14 & 146 \\
\hline adnormal changes in chest CT & 78 & 55 \\
\hline without clinical symptoms & 54 & $5.33 \pm 4.36$ \\
\hline $\begin{array}{l}\text { The media time from clinical } \\
\text { symptoms to NCP diagnosis (d) }\end{array}$ & $5.60 \pm 3.58$ & 0 \\
\hline Health care worker (n) & 0 & \\
\hline
\end{tabular}

\section{Discussion}

The disease of NCP got spread fast in Zhejiang province. The first patient, who came back from the city of Wuhan (Wuhan is the provincial city of Hubei province), was confirmed on January 20 in Zhejiang province. While there were 537 confirmed patients of NCP on January 30 in Zhejiang province, if this rate of disease spread hold on, the number of confirmed NCP patients might be more significant than 10 thousand on February 8 in Zhejiang province.

There were 1075 confirmed NCP patients on February 8 in Zhejiang province. The emergency measures of the Zhejiang province government had played a role in this result $\mathbb{1}$. restrict the movement of people extreme (it contained that forbidding the people into the Zhejiang province, restricting the local people to go outside); 2.send all the suspected NCP people to the hospital for further detection and isolate all the people who had contact with the confirmed or suspected NCP people in the past 14 days. According to the experience of SARS and MERS ${ }^{(9,10)}$, those measures were very necessary to prevent the NCP diffusing. And our data also proved those measures effectiveness: the number and the percentage of imported NCP patients got decreased quickly, and the number of new daily confirmed NCP patients also 
got smaller. Prof Zhao had pointed out the basic reproductive number $\left(R_{0}\right)$ of NCP ranged from 2.24 to $3.58^{(4)}$. And prof Li had pointed out the mean incubation period of NCP was 5.2 days, the epidemic doubled time of NCP was 7.4 days, and the $R_{0}$ of NCP was $2.2^{(3)}$. If we used 5 days as an incubation period, the real $R_{0}$ of NCP was smaller than 1 since January 31 in Zhejiang province. And the value R0 of NCP was 0.03 on February 8 (the number of newly confirmed NCP patients was 27 on February 8 , while the number of accumulation confirmed NCP patients was 829 on February 3 ).

What is the next challenge for preventing the NCP from spreading? Firstly, the NCP patients with unknown epidemiological history need to pay more attention. Those patients stood for the invisible and hidden spreading of NCP. Secondly, the super spreader is a significant threat to the Zhejiang province. And we did not forget the lessons of SARS ${ }^{(11,12)}$. we should maintain vigilance for the super spreader of NCP. Thirdly, the hospital infection is another critical point. And if the health care worker gets the infection, the situations stand for the hospital infection happening. In the announcement of the Wuhan health commission on January 21, there were 15 health care workers with confirmed NCP and 1 health care worker with suspected NCP. At another research of 138 confirmed NCP patients, 57 patients were presumed to get infected in the hospital. 40 of 57 patients were health care workers $\left({ }^{13)}\right.$. There was no health care worker with confirmed or suspected NCP in Zhejiang province till February 8 , and we can't ignore the risk.

What is the problem for the NCP's detection at present? Some researchers suggested us to use chest CT for early diagnosis criteria. Prof Xie pointed out that the NCP patients had typical imaging findings, including the ground-glass opacity in chest $\mathrm{CT}^{(14)}$. And the staff doctors can get the results half an hour after the patients had finished the chest $\mathrm{CT}$. While the PR-PCR assay would take at least one day to get the results. And the results of the PR-PCR assay might be a false negative. To prevent false-negative effects, we had to test at least two times of PR-PCR assay for suspected NCP patients ${ }^{(15)}$. And our data showed that average patients need more 5 days from having the symptom of confirming the NCP. Although we still used the positive results of PR-PCR assay or matching genetic sequence as the detection criteria, we need more efficient diagnosis criteria and methods.

Fever and cough were the most common early clinical symptoms for NCP patients in our research. Similar results also appeared in other studies $(16,17)$. The fatality rate in Zhejiang province was much lower. There were 1075 confirmed NCP patients in Zhejiang province without one patient dead on February 8. At the same time, there were 21700 established NCP patients in Hubei province with 780 dead patients. In the research of 99 NCP patients, the results reminded us that 17 patients developed acute 
respiratory distress syndrome, and 11 of $17 \mathrm{NCP}$ patients died of multiple organ failure ${ }^{(18)}$. And the reason for the differences in the fatality rate was worthy of further study.

At present, the government restricted the movement of people to prevent the NCP spread. At the same time, the measures made people's life inconvenient, and the economy paid a high price. And we did not know how long the measures would last. Moreover, we neither see the spread of NCP would appear again or not if the measures were canceled one day.

\section{Conclusion}

The early spread of NCP in Zhejiang province was speedy. After the government of Zhejiang province took strict measures to restrict the movement of people, the serious situation of NCP got noticeable relief in Zhejiang province since February 1. The potential risk of NCP in Zhejiang province was huge, and especially the government takes off the restriction of the movement of people in the future.

\section{Declarations}

\section{The statement}

we confirmed that our study was carried out in accordance with relevant guidelines.

\section{The conflict of interest}

no conflict of interest exists in the submission of this manuscript, and the manuscript is approved by all authors for publication. We want to declare on behalf of my co-authors that the work described was original research that has not been published previously, and not under consideration for publication elsewhere, in whole or in part.

\section{The authors contributions}

Writing the paper: Shen junwen

Collecting the data: Fang zihai, Yao jianxiang, Liu bingyao, Zhou ying, shi zhanqing Polishing the language: Chen yu, Tang jianer

Editing the paper: Wang rongjiang

\section{Funding information}

The medical and health platform of Zhejiang province (2020385100) 
Huzhou Science and Technology Bureau in 2018 (2018GYB12)

The medical and health platform of Zhejiang province (platform focus)(2016ZDB012)

\section{Reference}

1. Wu JT, Leung K, Leung GM. Nowcasting and forecasting the potential domestic and international spread of the 2019-nCoV outbreak originating in Wuhan, China: a modelling Lancet (London, England). 2020.

2. Zhu N, Zhang D, Wang W, et al. A Novel Coronavirus from Patients with Pneumonia in China, 2019. The New England journal of medicine.

3. Li Q, Guan X, Wu P, Wang X, et al. Early Transmission Dynamics in Wuhan, China, of Novel Coronavirus-Infected Pneumonia. The New England journal of medicine.

4. Zhao S, Lin Q, Ran J, et al. Preliminary estimation of the basic reproduction number of novel coronavirus (2019-nCoV) in China, from 2019 to 2020: A data-driven analysis in the early phase of the outbreak. International journal of infectious diseases : IJID : official publication of the International Society for Infectious Diseases.

5. Huang C, Wang Y, Li X, et al. Clinical features of patients infected with 2019 novel coronavirus in Wuhan, China. The Lancet.

6. Holshue ML, DeBolt C, Lindquist S, et First Case of 2019 Novel Coronavirus in the United States. The New England journal of medicine. 2020.

7. Rothe C, Schunk M, Sothmann P, et al. Transmission of 2019-nCoV Infection from an Asymptomatic Contact in Germany. The New England journal of medicine.

8. Patel A JD-nCRT. Initial Public Health Response and Interim Clinical Guidance for the 2019 Novel Coronavirus Outbreak. MMWR Morb Mortal Wkly Rep. 2020;69(5):140-6.

9. Graham RL, Donaldson EF, Baric RS. A decade after SARS: strategies for controlling emerging coronaviruses. Nat Rev Microbiol. 2013;11(12):836-48.

10. Wong G, Liu W, Liu Y, et al. MERS, SARS, and Ebola: The Role of Super-Spreaders in Infectious Disease. Cell Host Microbe. 2015;18(4):398-401.

11. Donnelly CA, Ghani AC, Leung GMea. Epidemiological determinants of spread of causal agent of severe acute respiratory syndrome in Hong Kong. The Lancet. 2003;361(9371):1761-6.

12. Leung GM HA, Ho LM, et al. The epidemiology of severe acute respiratory syndrome in the 2003 Hong Kong epidemic: an analysis of all 1755 patients. Ann Intern Med. 2004;141(9):662-73.

13. Wang D, Hu B, Hu C, et al. Clinical Characteristics of 138 Hospitalized Patients With 2019 Novel Coronavirus-Infected Pneumonia in Wuhan, China. JAMA.

14. Xie X ZZ, Zhao W, et al. Chest CT for Typical 2019-nCoV Pneumonia: Relationship to Negative RTPCR Testing. 2020;[Epub ahead of print]. 
15. Lin C, Ye R, Xia YL. A meta-analysis to evaluate the effectiveness of real-time PCR for diagnosing novel coronavirus infections. Genetics and Molecular Research. 2015;14(4):15634-41.

16. Lu R, Zhao X, Li J, Niu P, et al. Genomic characterisation and epidemiology of 2019 novel coronavirus: implications for virus origins and receptor binding. The Lancet.

17. Wang W, Tang J, Wei Updated understanding of the outbreak of 2019 novel coronavirus (2019-nCoV) in Wuhan, China. Journal of medical virology. 2020.

18. Chen N, Zhou M, Dong X, et al. Epidemiological and clinical characteristics of 99 cases of 2019 novel coronavirus pneumonia in Wuhan, China: a descriptive The Lancet. 2020.

\section{Figures}

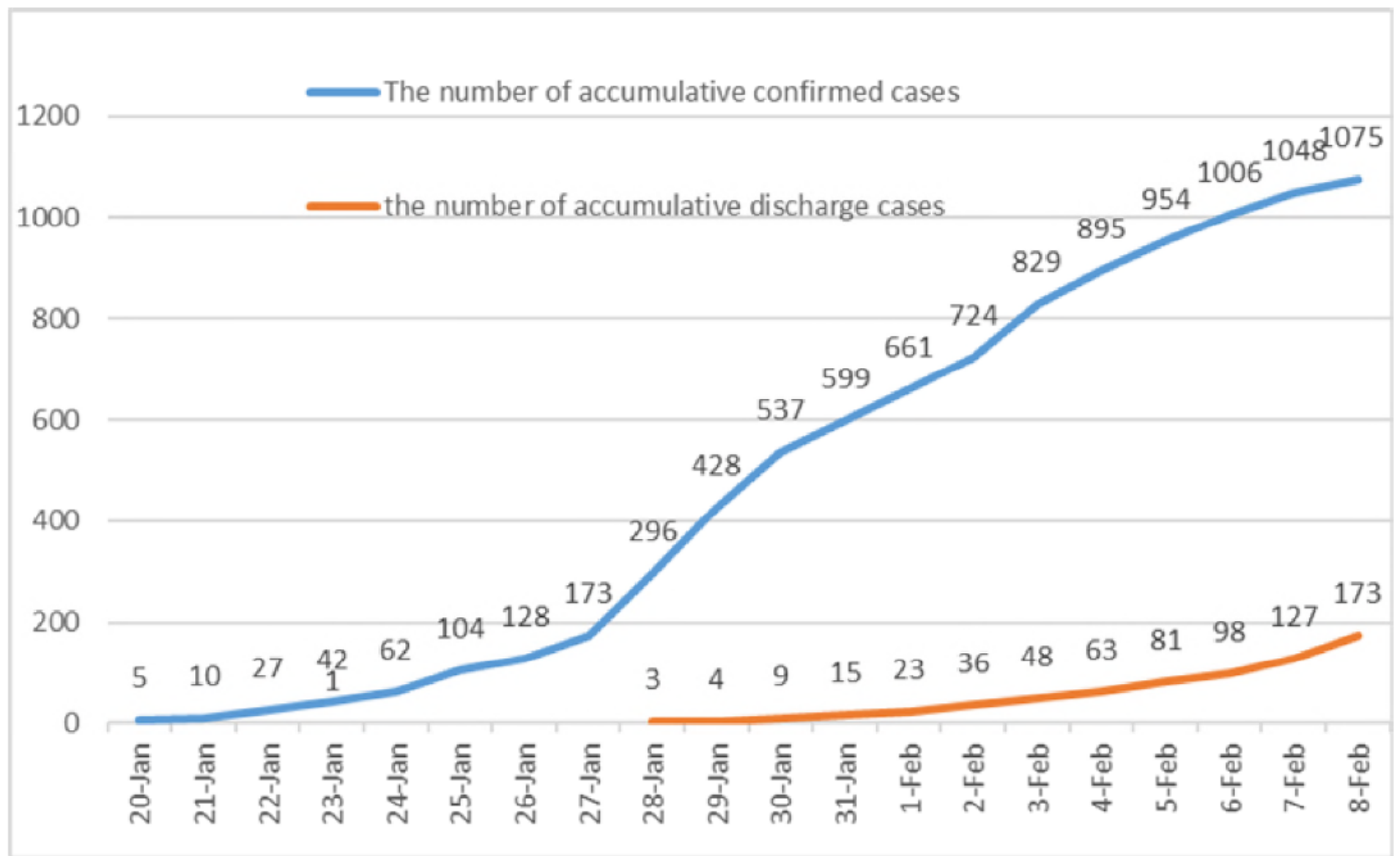

\section{Figure 1}

the number of accumulative confirmed NCP patients (blue line) and accumulative discharge NCP patients (orange line). 


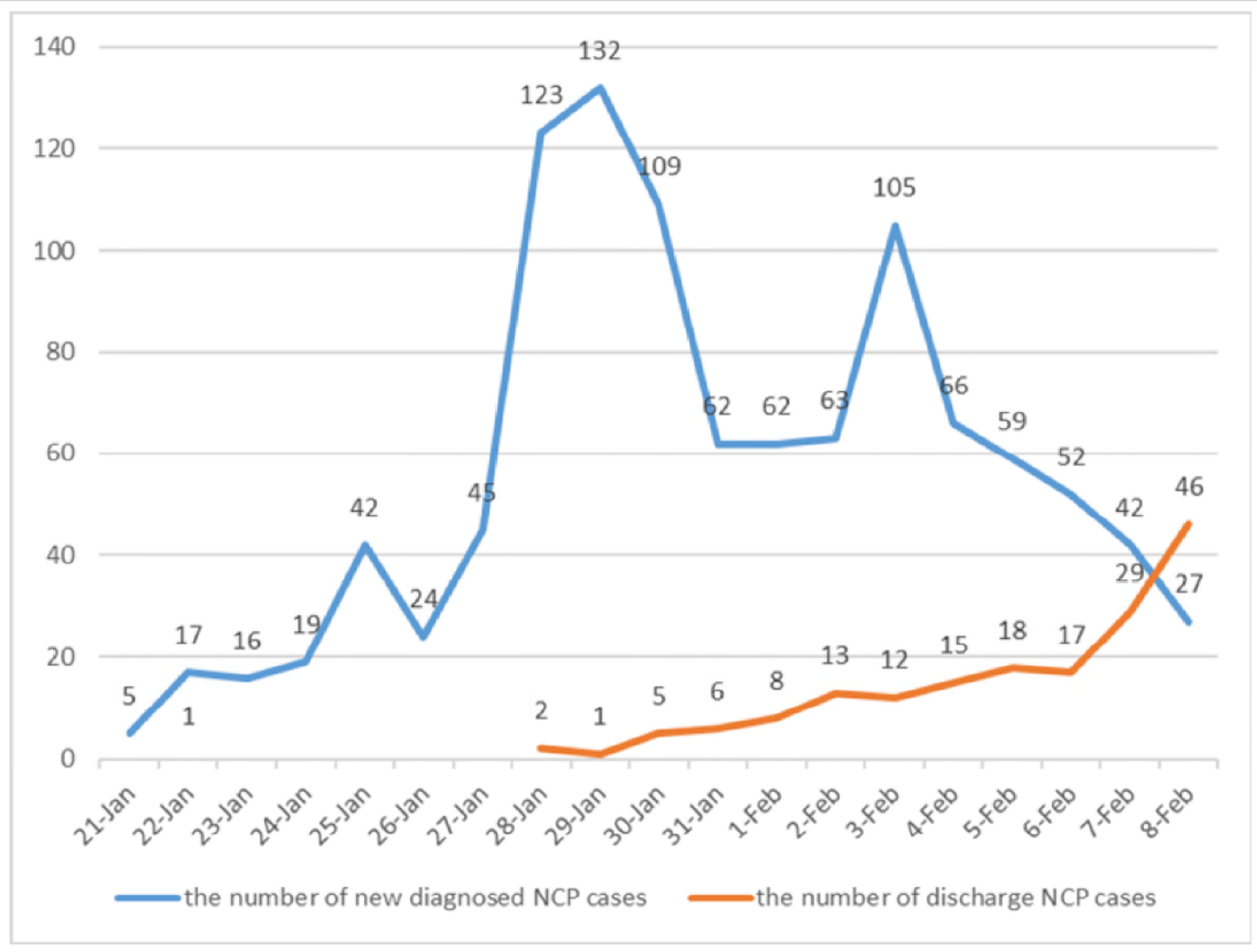

\section{Figure 2}

the daily number of new diagnosed NCP patients (blue line) and the daily number of discharge NCP patients (orange line). 


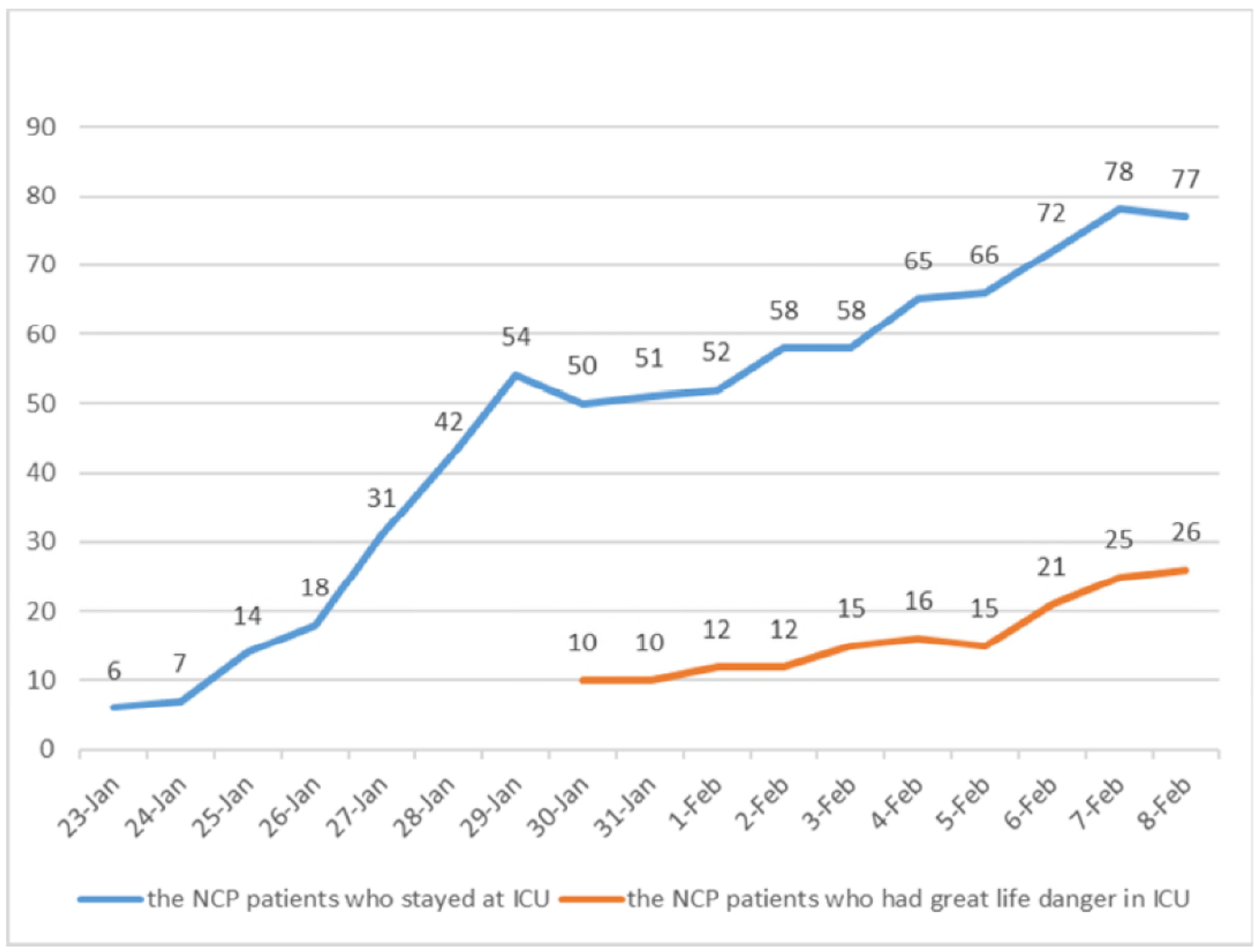

\section{Figure 3}

the number of accumulative NCP patients who stayed at ICU (blue line), and the number of accumulative NCP patients who had greater life danger in ICU (orange line). 


\section{epidemiological history}

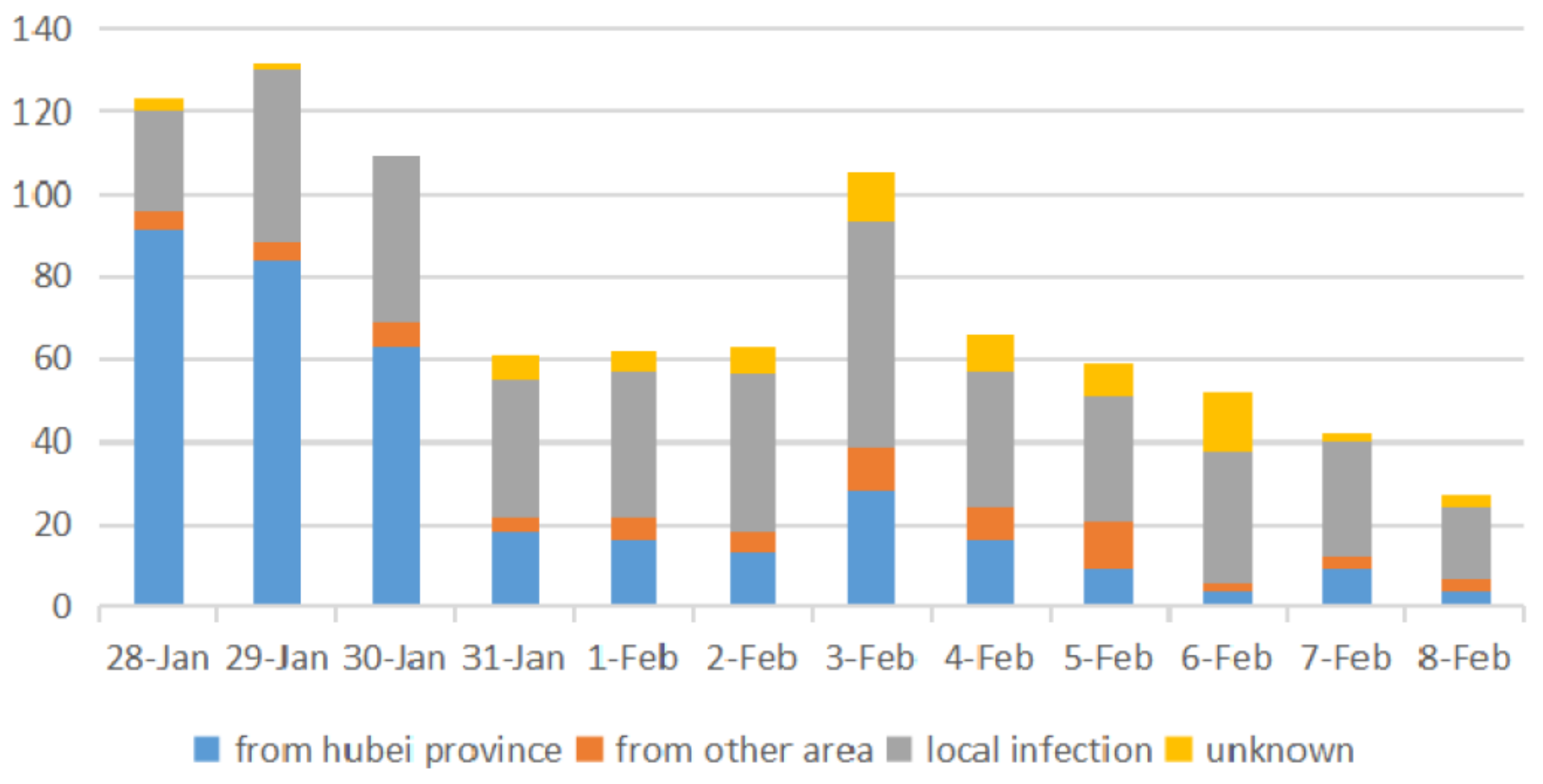

Figure 4

the daily number of new diagnosed NCP patients' epidemiological history 


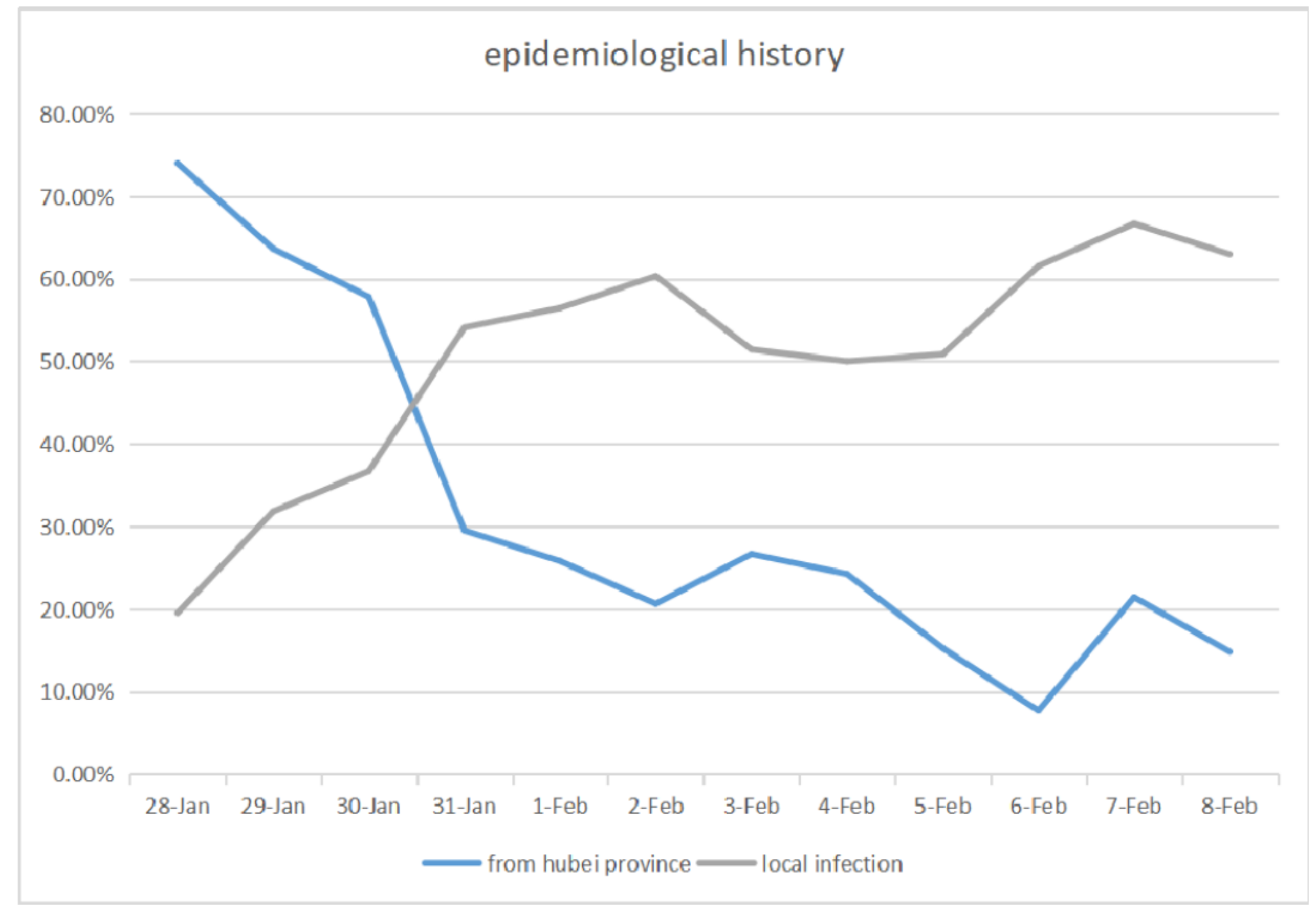

\section{Figure 5}

we compared the daily percentage of new diagnosed NCP patients who travelled from hubei province or got infected in local area. 


\section{epidemiological history}

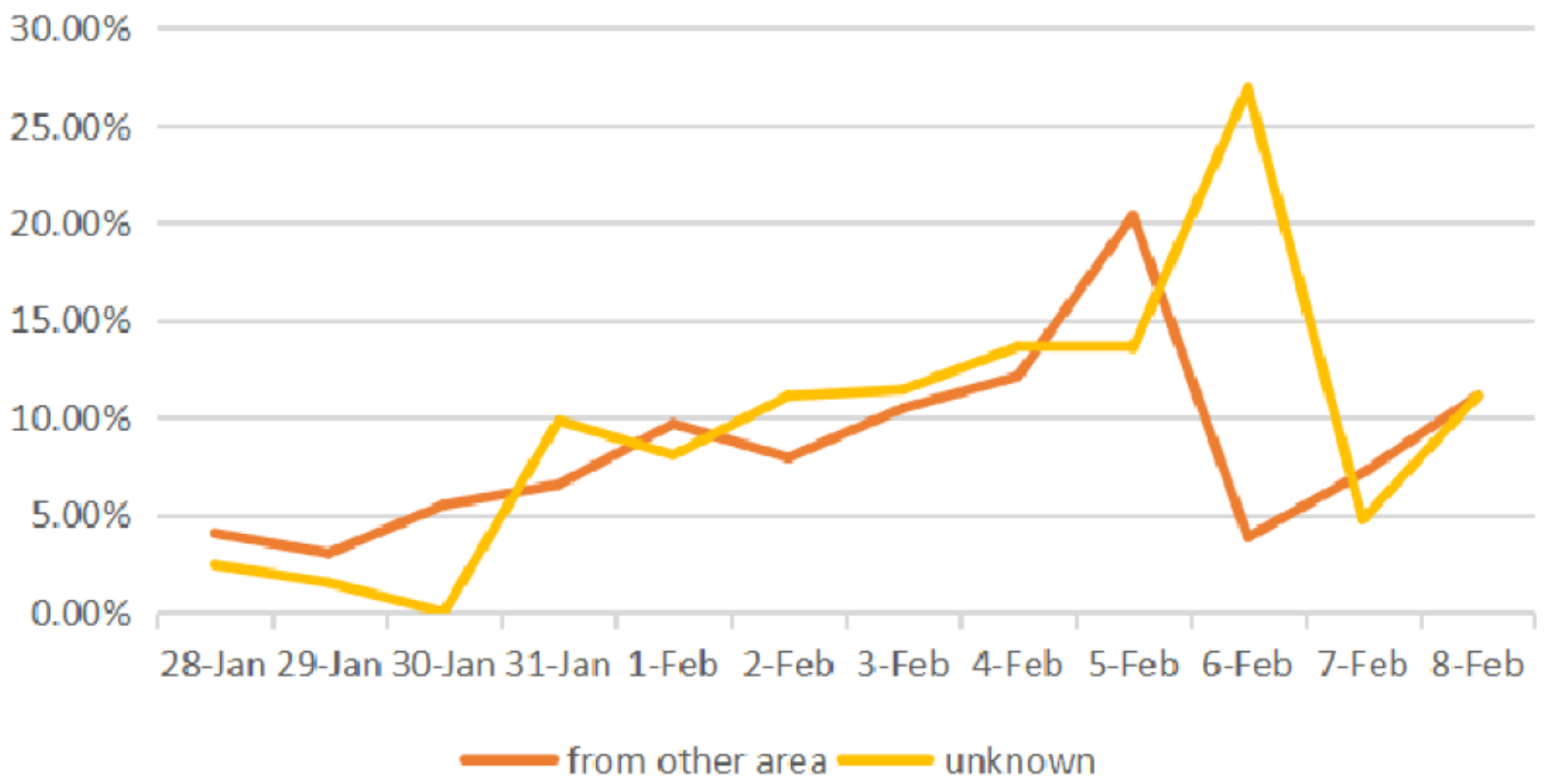

\section{Figure 6}

the daily percentage of new diagnosed NCP patients who travelled from other area not hubei province or with unknown epidemiological history.

\section{Supplementary Files}

This is a list of supplementary files associated with this preprint. Click to download.

- Supplementaltables.pdf 